\title{
Gold's evolving role in the electronics industry
}

\author{
Trevor Keel
}

Published online: 16 August 2014

(C) The Author(s) 2014. This article is published with open access at SpringerLink.com

Welcome to the third issue of Gold Bulletin in 2014. As usual, the manuscripts touch on diverse areas of science and technology, from historic methods of nanoparticle synthesis through to the continued relevance of new gold alloys to materials science.

One thing that the third issue's line up of papers did remind me of is the ongoing importance of gold in the electronics industry. In 2013, 280 tonnes of gold was used in total, primarily in the form of thin bonding wire and coatings. Practically, every item containing electronic components will utilise a small quantity of gold, from consumer goods such as smartphones and tablets, through to a broad range of critical systems in aeroplanes and cars. The fact of the matter is that we all use gold on a daily basis, and $99 \%$ of the population are completely unaware of it!

One conversation that has been prominent in the industry recently has been what is the future for gold in electronics. The last decade has seen the price of gold rise significantly, which has seen certain cheaper substitutes begin to gain traction. This has been particularly pronounced in the bonding wire sector, with copper and palladium-coated copper wires becoming increasingly commonly used in some applications. However, gold continues to be the material of choice in high-end and safety critical applications, and this is unlikely to change in the short term. There seems to be less evidence of substitution pressures in the use of gold salts for coatings in the industry, and even if there are, elements of this will be offset by the relatively buoyant semiconductor sector we are currently experiencing.
Whilst there are pressures, there are also great opportunities for the future. One area of real excitement is in flexible and stretchable electronics. As a material, gold lends itself perfectly to these types of applications, particularly when the device needs to come into contact with the human body in some way. As most people know, gold will not corrode or cause any unwanted side effects in vivo. Companies such as MC10 in Boston (http:// www.mc10inc.com/) are exploiting gold wires in some of their medical devices, and Google and Novartis recently announced (http://www.novartis.com/newsroom/mediareleases/en/2014/1824836.shtml) their intention to further develop and commercialise smart contact lenses which also rely on gold wiring for their unique functionality. There are also a number of other areas of promise, including highdensity data devices and new piezoelectrics.

Gold's unique properties have made it a critical material in the electronics industry and this relationship is set to continue into the future. Here at Gold Bulletin we continue to welcome any interesting manuscript on the subject, and encourage you all to spread the word about the journal with your colleagues and peers.

Enjoy the third issue of 2014 and best wishes as we move towards the end of the year.

Open Access This article is distributed under the terms of the Creative Commons Attribution License which permits any use, distribution, and reproduction in any medium, provided the original author(s) and the source are credited.

T. Keel $(\bowtie)$

World Gold Council, London, UK

e-mail: trevor.keel@gold.org 